\title{
The Bone Disease of Preterm Birth: A Biomechanical Perspective
}

\author{
MARVIN E. MILLER \\ Department of Pediatrics, Wright State University School of Medicine, Dayton, OH 45404, U.S.A.
}

\begin{abstract}
ABST
The bone disease of preterm birth has traditionally been
explained by a decrease in bone formation from insufficient
availability of calcium and phosphorus. However, there is emerg-
ing evidence that there is increased bone resorption in the bone
disease of preterm birth, an observation that indicates some other
explanation for this condition. The biomechanical model of
postnatal bone formation states that, through a regulatory feed-
back system in the bone called the mechanostat, bone is able to
respond to increased bone loading by increasing bone strength
and to decreased bone loading by decreasing bone strength. It is
suggested that this increased bone resorption in the markedly
preterm infant compared with the term infant is secondary to
\end{abstract}
decreased bone loading. Application of this model to the fetus and preterm infant suggests that intrauterine bone loading of the fetus from movement and kicking against the uterus is critical for normal fetal bone formation. The associated muscle growth from this activity also contributes to bone loading. The markedly preterm infant is deprived of much of this critical time period of intrauterine bone accretion, and bone formation occurs in the less favorable extrauterine environment, where there is significantly less bone loading. (Pediatr Res 53: 10-15, 2003)

Abbreviation
VLBW, very low birth weight
Infants who are born at 24, 25, and 26 wk of gestation now have a $50 \%, 70 \%$, and $80 \%$ likelihood for survival, respectively (1). This increasing survival rate of VLBW (VLBW = birth weight $<1500 \mathrm{~g}$ ), preterm infants over the past $30 \mathrm{y}$ has created new medical diseases in these fragile infants that did not previously exist. One such disease is the bone disease of preterm birth in which approximately $10 \%$ of VLBW, preterm infants incur fractures within the first several months of life. The mean age of diagnosis of fractures in one series of preterm infants with fractures was $76 \mathrm{~d}$, and the types of fractures included long bone, rib, and metaphyseal fractures (2). The specific cause of the bone disease of preterm birth must explain the observation that the rate of bone accretion for a fetus in an intrauterine environment is greater than that for a preterm infant in an extrauterine environment. Thus, the bone density of a full-term infant who is born at $40 \mathrm{wk}$ of gestation is greater than that of a 12-wk-old preterm infant who is born at $28 \mathrm{wk}$ of gestation (3).

It has been assumed that this difference in bone accretion rates in the intrauterine versus the extrauterine environment is from the difference in availability of calcium and phosphorus,

Received July 17, 2001; accepted August 22, 2002

Correspondence: Marvin Miller, M.D., Children's Medical Center, Department of Medical Genetics, 1 Children's Plaza, Dayton, OH 45404, U.S.A.; e-mail: srmmem@aol.com

Supported, in part, by the Children's Medical Center Research Foundation, Dayton, OH, U.S.A.

DOI: 10.1203/01.PDR.0000039922.25000.17 the essential minerals needed for bone formation (4). There is an exponential increase in bone formation during the last trimester during which approximately $80 \%$ of fetal bone is produced as the whole-body calcium increases from approximately $5 \mathrm{~g}$ at $24 \mathrm{wk}$ of gestation to approximately $30 \mathrm{~g}$ at term, 40 wk of gestation (5). The peak accretion rate for bone occurs at approximately $35 \mathrm{wk}$ of gestation, when the calcium accretion rate is approximately $150 \mathrm{mg} / \mathrm{kg} / \mathrm{d}$ and the phosphorus accretion rate is approximately $75 \mathrm{mg} / \mathrm{kg} / \mathrm{d}$. It is interesting that there is a dramatic decrease in calcium accretion in the last 5 wk of pregnancy (6). For adequately meeting this large demand for calcium and phosphorus during this rapid period of bone formation during the last trimester, there is active transplacental transport of calcium and phosphorus from the mother to the fetus (7). It has been assumed that for normal extrauterine bone formation in a VLBW, preterm infant, similar daily requirements of calcium and phosphorus are needed. However, in the extrauterine environment, it is difficult to achieve this level of calcium and phosphorus delivery in the VLBW, preterm infant with enteral formulas or hyperalimentation (8). The cause of the bone disease in preterm birth has been ascribed to this particular issue of mineral substrate availability. Contributing factors to the bone disease of preterm birth include chronic illness, prolonged hyperalimentation, bronchopulmonary dysplasia, and the use of hypercalciuric drugs such as furosemide for treatment of bronchopulmonary dysplasia and methylxanthines for treatment of apnea and bradycardia, both of which increase calcium losses (8). 


\section{INCREASED BONE RESORPTION IN THE BONE DISEASE OF PRETERM BIRTH}

Although the notion that decreased bone formation from diminished availability of calcium and phosphorus has traditionally been thought to be the primary cause of the bone disease of preterm birth, there are two observations that suggest otherwise and provide evidence of increased bone resorption in the bone disease of preterm birth. First, two separate groups have found urinary bone resorption markers in much greater concentration in preterm infants compared with term infants $(9,10)$. Markers of bone resorption included collagen turnover compounds (hydroxyproline, type 1 collagen telopeptide), calcium, and phosphate. Beyers et al. (9) noted that preterm infants at expected full-term age had significantly greater urine excretion of calcium $(2.9 \times)$, phosphate $(4.3 \times)$, and hydroxyproline $(3.7 \times)$ compared with normal term infants. Serum alkaline phosphatase was twice as great $(411 \mathrm{U} / \mathrm{L}$ versus $206 \mathrm{U} / \mathrm{L}$ ) in preterm infants at expected full-term age compared with the normal term infants. Moreover, radiologic evaluation showed increased endosteal resorption in the preterm infants. Mora et al. (10) found that preterm infants (average gestational age of $33 \mathrm{wk}$ ) had significantly higher blood levels of type 1 collagen telopeptide than term infants when both groups were studied at 4 wk of age. Using osteocalcin and procollagen type 1 carboxyterminal propeptide as indices of bone formation, these investigators found lower levels of bone formation in the preterm infants than the term infants. Others have also found an increased renal excretion of calcium and phosphorus in the preterm infant compared with the term infant (11).

Second, VLBW, preterm infants who are fed formula preparations with higher calcium and phosphorus content usually do not have an increased bone density (12). If the bone disease of preterm birth were caused solely by calcium and phosphorus deficiency, then these two observations would not be expected. The totality of evidence points to some other factor or additional factor than just calcium and phosphorus availability as the basis for the bone disease of preterm birth.

What specific factor could explain this difference in bone resorption in the preterm versus term infant? In the past, a biochemical perspective has been taken in trying to understand human bone disease as shown in Figure 1A. This approach has failed to provide adequately an understanding of the bone disease of preterm birth, because it ignores biomechanical factors. If a biomechanical perspective is taken, then the bone disease of preterm birth is easily understood as shown in Figure 1B. The answer to this conundrum is in the difference in bone loading of the skeletal system in the preterm infant compared with the term infant.

\section{BIOMECHANICAL MODEL OF BONE FORMATION}

Frost (13) proposed the mechanostat/mechanical loading model of postnatal bone formation, which states that the primary factor in the development of bone strength is the load (force) placed on the bone. The load causes a strain on the bone, which is transmitted to the mechanostat as an input signal. The mechanostat is a sensor within the bone that can
A. Biochemical Model of Bone Formation

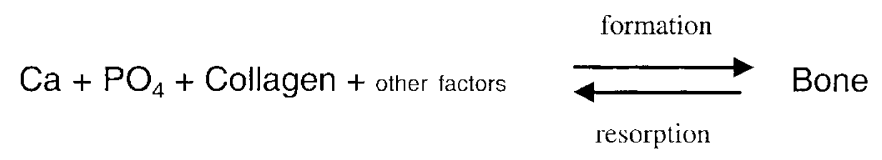

\section{B. Biochemical/Biomechanical Model of Bone Formation (simplified version of the "Utah paradigm")}

\section{Bone Loading}

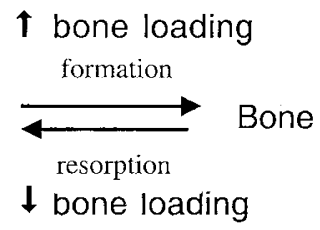

Figure 1. Models of bone formation. A, Biochemical model of bone formation. The biochemical model of bone formation recognizes that bone resorption and formation are mediated by a number of biochemical and hormonal parameters, including calcium, phosphorous, collagen, and others (e.g. vitamin D, PTH, alkaline phosphatase). However, this model does not provide an explanation for the primary impetus for bone formation and resorption. $B$, Biochemical/biomechanical model of bone formation (Utah paradigm). The biochemical/biomechanical model is similar to the above biochemical model with the important addition of the influence of bone loading. Bone loading is the mechanical catalyst that drives the above reaction either to form bone if there is increased bone loading or to resorb bone if there is decreased bone loading. Bone loading is far greater in a fetus in the intrauterine environment than in a newborn infant in the extrauterine environment because of the ability of the fetus to kick against the uterine wall in the buoyancy of the amniotic fluid. Fetal activity also promotes muscle growth, which contributes to bone loading.

evaluate the input of strain from a given load placed on the bone and then direct an appropriate output to the effector cells, osteoblasts and osteoclasts. Strain is the proportional change in length caused by a load and can be from compression, tension, or shearing loads. If a bone specimen is stretched by $1 \%$ of its length, then it is said to be undergoing a strain of $1 \%$, or 10,000 microstrain. If a bone specimen is compressed by $0.1 \%$ of its original length so that it is now $99.9 \%$ of its original length, then it would be undergoing a strain of 1000 microstrain. Loads always cause strains even when they are small.

The mechanostat thus is the "brain of the bone" and functions as a feedback or regulatory system to keep bone strength commensurate with the loads placed on the bone. The mechanostat processes the strain input and compares it with preset, threshold levels of strain for increasing bone strength or decreasing bone strength. The mechanostat is then able to generate an appropriate output signal to effector cells to bring about the needed change in bone strength to align the strain within given limits. Effector cells are osteoblasts that produce bone and osteoclasts that resorb bone. If significantly increased loads that exceed the threshold for increasing bone strength are placed on a bone, then the mechanostat signals the effector 
cells to increase bone strength. If significantly decreased loads that exceed the threshold for decreasing bone strength are placed on a bone, then the mechanostat signals the effector cells to decrease bone strength. If the strain does not exceed either of these thresholds, then the effector cells operate at a status quo, or baseline, level of activity.

Osteoblast and osteoclast activity can change bone strength through either altering bone density or altering bone architecture through two distinctly different processes of bone physiology: bone modeling and bone remodeling. Both modeling and remodeling are processes that respond to bone loading through the mechanostat.

Modeling is the process by which bone is sculpted to the most advantageous geometry, both for bone strength and for appropriate attachment of muscles and tendons. As bone grows, some bone must be added to certain surfaces and some bone must be removed from other surfaces. The bone achieves this result through formation drifts and resorption drifts. Formation drifts influence osteoblasts to build up some bone surfaces, whereas resorption drifts influence osteoclasts to remove bone from some bone surfaces. Modeling results in changes of bone size or shape or of both and thus is a prominent process in bone development during fetal life and in childhood. In bone modeling, osteoblasts and osteoclasts function independent of each other, and each cell type responds to a certain preset modeling threshold within the mechanostat. Modeling almost always increases bone strength either by increasing bone mass or by favorably altering bone architecture.

Remodeling is the process whereby fatigued bone is efficiently removed and then replaced by new, intact bone by the sequential activity of osteoclasts to resorb the fatigued bone followed by osteoblasts to produce new bone. In bone remodeling, osteoblasts and osteoclasts act cooperatively in a coupled manner in a unit called a basic multicellular unit. This coordinated activity is also realized when a certain preset threshold of strain is sensed by the mechanostat.

There are two types of bone loading. The first is associated with the direct contact or impact of bone against another object, such as the increased load that the leg bones realize during running or from the resistance that a bone might experience such as the extremities realize in swimming. The second is associated with the active and passive load that the bone senses from the muscles attached to it. The muscles that attach to a bone exert a small but continuing load on the bone even when the muscle is not actively moving the bone. The loading of the skeletal system from attached muscles is critical in maintaining bone density. Weightlifters have greater bone density than nonweightlifters (14). Children with chronic, neuromuscular diseases associated with muscle paralysis and muscle weakness have osteopenia and an increased risk for fracture (15).

Frost called this model the "Utah paradigm," which is an ever-evolving paradigm that includes nutrient, hormonal, cellular, biochemical, and biomechanical factors (16). The centerpiece of the Utah paradigm is the notion that bone strives to be a mechanically competent tissue through the operation of the mechanostat to sense strain from the loads placed on the bone and appropriately respond to these strains by increasing or decreasing bone strength. Others have also underscored the importance of mechanical considerations in skeletal health and disease (17-19).

According to the Utah paradigm, bones are formed in two distinct steps (16). The first step is the embryogenesis of the skeletal system. Between 5 and $12 \mathrm{wk}$ of gestation, multiple, specific genes direct condensations of mesenchyme to specific anatomic locations that are destined to become the precursor tissues of bone that will eventually chondrify and ossify (20). In a similar time period during the first trimester, other specific genes direct ventral and dorsal condensations of somitic mesoderm to become precursor tissues of skeletal muscle, and these will eventually attach to their appropriate bones. By 16 wk of gestation, the anatomy, anatomical relationships, and biologic machinery for adaptation of bones are in place. This state of bone is called the baseline conditions.

The second step of bone formation, beginning during the midportion of the second trimester, is the state of responsiveness of the skeletal system to genetically defined bone proteins and humoral mediators, nutrient considerations, and mechanical factors. Long bones grow both in length and in diameter. Linear bone growth of the long bones is determined primarily by specific genes through enchondral ossification. However, long bones assume their final, normal geometry, through the process of modeling. Modeling uses osteoblasts to form bone and osteoclasts to remove bone. Diametrical bone growth of long bones occurs through modeling in which osteoclasts remove bone from the endosteum and osteoblasts form bone along the periosteum. Flat bones, such as the scapula, pelvis, and skull, grow through intramembranous bone formation. Modeling that is responsive to bone loading occurs in enchondral and intramembranous bone formation in both the prenatal and postnatal periods. During the second and third trimesters, bone modeling also responds to increasing muscle forces. As the skeletal system of an individual ages, the adaptations for any given bone, which include growth, modeling, and remodeling, are added to the baseline conditions of the bone.

At the same time that bone modeling begins, fetal movement commences at approximately the 16th wk of gestation. Bone modeling is strongly influenced by bone loading, and bone loading during fetal life is determined primarily by fetal movement. Fetal movement leads to bone loading in three ways: 1 ) the loading associated with the impact of the fetus, especially the extremities, against the uterus; 2) the loading associated with the resistance against movement in the amniotic fluid; and 3) the loading associated with normal fetal muscle development, which is movement dependent.

The Utah paradigm, therefore, predicts that bone strength and, thus, bone density and bone architecture are directly related to fetal movement. In situations in which there is diminished fetal movement, decreased bone strength of the fetus and newborn is expected through changes in bone density and bone architecture. Three observations support the hypothesis that fetal movement determines fetal bone strength. First, previous work by Rodriguez et al. (21) showed that infants with congenital neuromuscular disease in which there is both decreased fetal movement and decreased fetal muscle mass and 
function have osteopenia and decreased cortical bone thickness of long bones compared with controls. This observation suggests that there is diminished subperiosteal bone formation in infants with prenatal-onset neuromuscular disease. Second, Rodriguez et al. $(22,23)$ also described an experimental animal model called the fetal akinesia deformation sequence in which rat fetuses are pharmacologically immobilized with curare at $17 \mathrm{~d}$ of gestation (term gestation is $21 \mathrm{~d}$ ). At birth, these curare-exposed rats have short umbilical cords and osteopenia compared with controls. Decreased fetal movement leads to a short umbilical cord (24). Third, diminished fetal movement and intrauterine confinement have been put forth as the underlying basis of temporary brittle bone disease $(25,26)$. This observation has suggested that prenatal bone loading in the form of fetal movement can influence postnatal bone strength during the first year of life and that infants who had significantly decreased fetal movement may be at risk for incurring fractures with physical forces that might not ordinarily cause a fracture, especially in the first 4 mo of life. Preterm birth is overrepresented in infants with temporary brittle bone disease (25).

\section{APPLICATION OF THE BIOMECHANICAL MODEL OF BONE FORMATION TO THE BONE DISEASE OF PRETERM BIRTH}

That the fetus has a functioning musculoskeletal system by $16 \mathrm{wk}$ of gestation sets the backdrop for the influence of biomechanics on fetal bone development after 16 wk of gestation. Whereas genetic information, hormonal influences, and nutrient considerations all influence bone physiology after 16 wk of gestation and into postnatal existence, it is biomechanical considerations that primarily determine ultimate bone strength, and these considerations have not been fully appreciated in the past in models of bone physiology before the Utah paradigm.

The extent of in utero bone loading will determine the ultimate skeletal strength of the fetus, especially during the last trimester, when there is rapid bone growth and bone mineralization. Fetal movement in the third trimester is the critical event that endows the newborn infant with normal bone loading and, thus, normal skeletal strength. The term infant who has an intact neuromuscular system realizes the full influence of this fetal movement on bone formation. The intrauterine loading of the fetal musculoskeletal system through fetal movement activates the mechanostat to increase bone strength through the process of modeling as shown in Fig. 2. Fetal movement also promotes muscle growth, which contributes to bone loading and thus also influences bone modeling.

When an infant is born markedly preterm, however, the infant is deprived of much of this musculoskeletal bone loading in utero as also shown in Fig. 2. After birth, the markedly preterm infant is often hypotonic and has a poverty of movements compared with the term infant (27). Attenuated bone loading in the VLBW, preterm infant leads to an input strain to the mechanostat that is lower compared with the term infant. Thus, there is also postnatal modulation of the mechanostat to increase resorption and decrease bone formation in the VLBW, preterm infant compared with the term infant. The markedly preterm, VLBW infant is, therefore, at a distinct biomechanical disadvantage in bone formation by losing weeks of meaningful intrauterine movement that promotes bone formation and replacing this period with that of an earlier-than-expected encounter with the extrauterine environment, which is less favorable for bone formation.

The rib fractures associated with preterm birth have the same underlying cause as the long bone fractures. The bone loading of ribs probably can occur from the following: 1) fetal movement and kicking that likely is transmitted along the skeleton to the ribs; 2) active or passive breathing, which would provide some bone loading through the ribs expanding in inspiration and contracting in expiration; and 3) the muscles attached to the ribs, which indirectly would get stronger with active breathing but probably would not get stronger if breathing were assisted by mechanical ventilation. Thus, a term infant who benefited from the loading of the skeleton during the entire 40 wk of gestation and who was actively breathing and not ventilator dependent during the newborn period would have stronger ribs than the 28 -wk preterm infant who was deprived of $12 \mathrm{wk}$ of exuberant intrauterine bone loading and who was on a ventilator for a prolonged period after birth because of respiratory problems. Rodriguez et al. (21) found that the periosteal diameter of the fifth rib in infants with prenatal-onset neuromuscular disease was significantly lower than that of control infants, which indicates that prenatal bone loading does influence rib strength.

The recently published study by Moyer-Mileur et al. (28) provides support that bone loading is important in bone formation of the preterm infant. They found that preterm infants who received daily physical therapy for an average period of approximately $27 \mathrm{~d}$ after birth had gains in forearm bone mineral density that were $75 \%$ greater than those in infants who did not receive physical therapy. This daily physical activity mimics the movement that this born, preterm infant would have had as an intrauterine fetus if the infant had not been born preterm.

The traditional, biochemical paradigm of bone biology adequately details the intricate relationships between calcium, phosphorous, and various other hormonal influences on their disposition, as shown in Fig. 1A. However, the biochemical model does not consider the primary determinant of bone formation, bone loading. Only when biomechanical factors are considered in the context of the biochemical model of bone biology is there a complete picture of bone dynamics as shown in Figure 1B. This biomechanical model of perinatal bone formation suggests that mineral availability needs to be matched for the degree of bone loading on the skeletal system of the fetus/infant. Intrauterine movement of the fetus in the last trimester is much greater than the movement of the newborn infant, term or preterm, in the immediate postnatal period. The intrauterine environment is unique and well-suited for promoting bone loading of the fetal musculoskeleton, for the fetus is buoyed in amniotic fluid, which allows for bouncing and kicking against the uterine wall. This type of environment cannot be duplicated once the infant is born; thus, the VLBW, preterm 
Term Infant

[40 weeks gestation]

\section{Biomechanical Processing}

Preterm Infant

[12 week old infant born at 28 weeks gestation]

\section{Relative to Term Infant:}

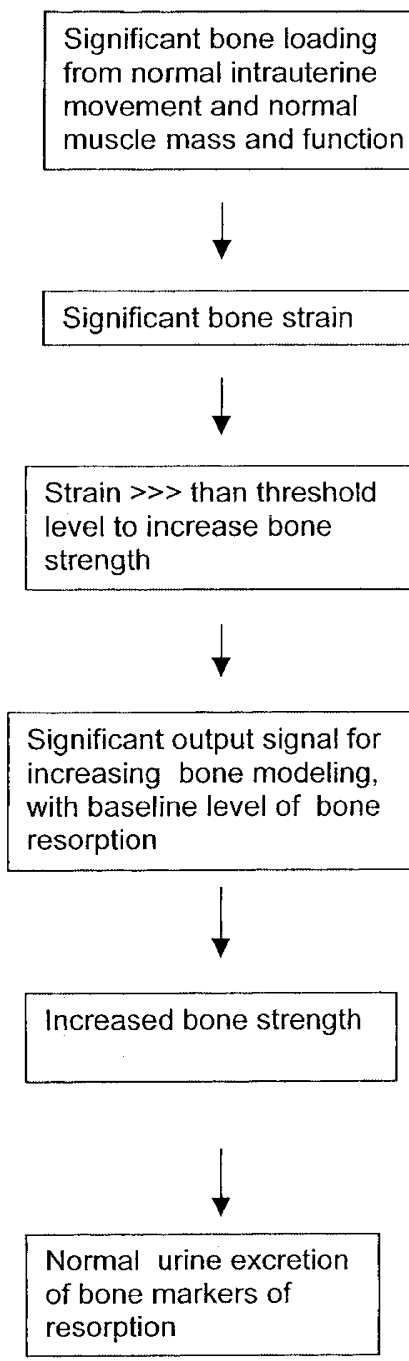

Bone Loading

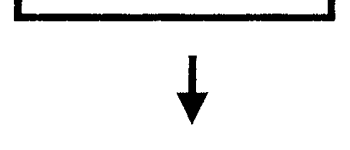

Strain on Bone

Input to mechanostat
Decreased bone loading from decreased intrauterine movement, decreased extrauterine movement, and decreased muscle mass and function

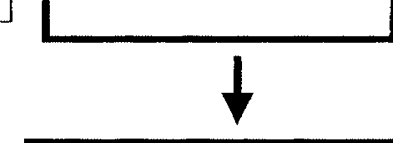

\section{.}

Mechanostat

Compares strain to preset, threshoid levels for increasing or decreasing bone strength

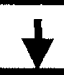

Signaling of effector cells Output from mechanostat

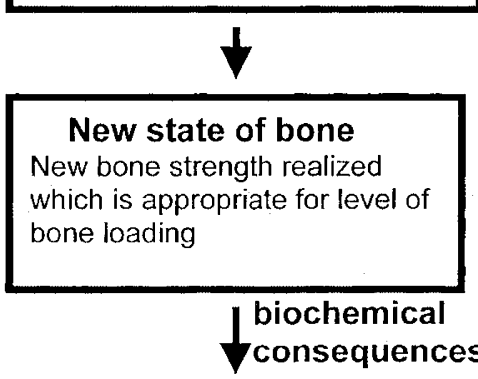

Urine excretion of bone markers of resorption:

calcium, phosphate, collagen turnover products
Increased urine excretion of bone markers of resorption
Strain > threshold level to increase bone strength

Less output signal for bone modeling with increased bone resorption

Less of an increase in bone strength

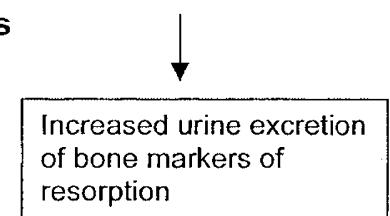

Figure 2. Biomechanical processing of bone loading in the VLBW, preterm infant $v s$ the term infant.

infant loses this critical period of intrauterine bone formation.

This model predicts that the amount of mineral needed for bone formation would be less once the infant is born, because of the change in the quality and quantity of movement, yet it has been the goal to attain in the VLBW preterm infant mineral availability that is comparable to the in utero delivery of calcium and phosphorus. The conventional wisdom of trying to achieve the same levels of mineral availability in the immediate postnatal period should be reevaluated for it may not be physiologically adaptive to the level of bone loading. The lower concentrations of calcium and phosphorus in breast milk may be giving us this message.

The biomechanical model of bone formation also explains the observations that chronic illness, bronchopulmonary dysplasia, and hyperalimentation are associated with the bone disease of preterm birth. All of these are associated with relative immobilization compared with a healthy, term infant and therefore would lead to increased bone resorption. The decrease in calcium accretion between 35 and 40 wk of gestation also follows from the biomechanical paradigm, because there is a physiologic crowding of the fetus during this time, with a rapidly increasing fetal volume and decreasing amniotic fluid volume (29).

This biomechanical paradigm has important therapeutic implications in the treatment of the bone disease of preterm birth. In addition to the amounts of calcium and phosphorus in the diet of the VLBW, preterm infant, attention should be given to the bone loading of these infants. Passive rangeof-motion activities should be considered in these infants, the success of which has been demonstrated by MoyerMileur et al. (28). The frequency of nephrocalcinosis, which is associated with VLBW infants, might be decreased with this approach, as it would increase bone accretion and thus 
decrease the amount of calcium presented to the kidney (30).

\section{CONCLUSION}

Fetal bone accretion is directly related to fetal movement. Although nutritional factors such as mineral availability may be contributing factors in the bone disease of preterm birth, this condition is best explained by the decreased quality and quantity of intrauterine and extrauterine movement of the VLBW, preterm infant compared with that of the term infant. Some of the problematic issues of the bone disease of preterm birth and rational therapeutic interventions for its treatment become readily understandable with a biomechanical perspective of this issue.

Acknowledgment. The author thanks Dr. Thomas N. Hangartner and Shelley Miller for their suggestions and critical review of this manuscript.

\section{REFERENCES}

1. Lorenz JM 2000 Survival of the extremely preterm infant in North America in the 1990s. Clin Perinatol 27:255-262

2. Dabezies EJ, Warren PD 1997 Fractures in very low birth weigh infants with rickets. Clin Orthop 335:233-239

3. Greer R, McCormick 1986 Bone growth with low bone mineral content in very low birth weight preterm infant. Pediatr Res 20:925-928

4. Bishop NJ 1999 Metabolic bone disease. In: Rennie JM, Roberton NRC (eds) Textbook of Neonatology, 3rd ed. Churchill Livingstone, London, pp 1002-1008

5. Rigo J, De Curtis M, Pieltain C, Picaud JC, Salle BL, Senterre J 2000 Bone mineral metabolism in the micropremie. Clin Perinatol 27:147-170

6. Forbes GB 1976 Calcium accumulation by the human fetus. Pediatrics 57:975-976

7. Care AD 1996 Unique aspects of calcium and vitamin D metabolism in the placenta and fetus. In: Gluckman PD, Heymann MA (eds) Bone and Cartilage. Pediatrics and Perinatology: The Scientific Basis. Arnold, London, pp 540-542

8. Demarini S, Mimouni FB, Tsang RC 1997 Disorders of calcium, phosphorus, and magnesium metabolism. In: Fanaroff AA, Martin RJ (eds) Neonatal-Perinatal Medicine: Diseases of the Fetus and Infant, 6th ed. Mosby, St. Louis, pp 1473
9. Beyers N, Alheit B, Taljaard JF, Hall JM, Hough SF 1994 High turnover osteopenia in preterm infants. Bone 15:5-13

10. Mora S, Weber G, Bellini A, Bianchi C, Chiumello G 1994 Bone modeling alteration in preterm infants. Arch Pediatr Adolesc Med 148:1215-1217

11. Karlen J, Aperia A, Zetterstrom R 1985 Renal excretion of calcium and phosphate in preterm and term infants. J Pediatr 106:814-819

12. Farek J, Petersen S, Peitersen B, Michaelsen KF 2000 Diet and bone mineral content in term and preterm infants. Pediatr Res 47:148-156

13. Frost HM 1996 Perspectives: a proposed general model of the "mechanostat" (suggestions from a new paradigm). Anat Rec 244:139-147

14. Conroy BP, Kraemer WJ, Maresh CM, Fleck SJ, Stone MH, Miller PD, Dalsky GP 1993 Bone mineral density in elite junior Olympic weightlifters. Med Sci Sports Exerc 25:1103-1109

15. Larson CM, Henderson RC 2000 Bone mineral density and fractures in boys with Duchenne muscular dystrophy. J Pediatr Orthop 20:71-74

16. Frost H 2001 From Wolff's law to the Utah paradigm: insights about bone physiology and its clinical applications. Anat Rec 262:398-419

17. Jee WSS 1989 The skeletal tissues. In: Weiss L (ed). Cell and Tissue Biology: A Textbook of Histology. Urba and Schwartzenberg, Baltimore, pp 211-259

18. van der Meulen MCH, Carter D, Beaupre GS 2001 Skeletal development: mechanical consequences of growth, aging, and disease. In: Marcus R, Feldman D, Kelsey J (eds) Osteoporosis, 2nd ed. Academic Press, London, pp 471-488

19. Rauch F, Schoenau E 2001 The developing bone: slave or master of its cells and molecules? Pediatr Res 50:309-314

20. Larsen WJ 1998 Essentials of Human Embryology. Churchill Livingstone, New York, pp 207-216

21. Rodriguez JI, Palacios J, Garcia-Alix A, Pastor I, Paniagua R 1988 Effects of immobilization on fetal bone development. A morphometric study in newborns with congenital neuromuscular diseases with intrauterine onset. Calcif Tissue Int 43:335339

22. Rodriguez JI, Palacios J, Ruiz A, Sanchez M, Alvarez I, Demiguel E 1992 Morphological changes in long bone development in fetal akinesia deformation sequence: an experimental study in curarized rat fetuses. Teratology 45:213-221

23. Rodriguez JI, Palacios J 1991 Pathogenetic mechanisms of fetal akinesia deformation sequence and oligohydramnios sequence. Am J Med Genet 40:284-289

24. Miller ME, Higginbottom M, Smith DW 1981 The short umbilical cord: its origin and relevance. Pediatrics 67:618-621

25. Miller ME, Hangartner TN 1999 Temporary brittle bone disease: association with decreased fetal movement and osteopenia. Calcif Tissue Int 64:137-143

26. Miller ME 1999 Temporary brittle bone disease: a real entity? Semin Perinatol 23:174-182

27. Kakebeeke TJ, von Siebenthal K, Largo RH 1997 Differences in movement quality at term among preterm and term infants. Biol Neonate 71:367-378

28. Moyer-Mileur LJ, Brunstetter V, McNaught TP, Gill G, Chan GM 2000 Daily physical activity program increases bone mineralization and growth in preterm very low birth weight infants. Pediatrics 106:1088-1092

29. Queenan JL 1991 Oligohydramnios and polyhydramnios. Contemp Obstet Gynecol $36: 60$

30. Adams ND, Rowe JC 1992 Nephrocalcinosis. Clin Perinatol 19:179-195 